\title{
Errrata
}

\section{Corrections to the article "Sharp Fronts of Paired Oscillatory Integrals"}

(Vol. 12, Suppl., 1977, pp. 53-68)

By

\section{Lars GÅRDING*}

It was pointed out to me by David Finch that my definition on p. 62 of Petrovsky chains and cycles for half-integral $q$ does not make sense, although Figure 2, illustrating these objects for $N=2$, is correct. I submit a corrected definition, replacing lines 4-19 of p. 62 .

2. Half-integral $q$. Let $Z$ be the two-sheeted cover of $\Omega \times Y$ consisting of all $\left(x, \theta, 0_{N}\right)$ with $(x, \theta)$ in $X \times \Omega$ and $\theta_{N}^{2}-\varphi(x, \theta)=0$. Let $U$ be an open part of $X \times \operatorname{Re} \Omega$ and construct $C^{\infty}$ chains $c(x):(\theta, t) \rightarrow$ $v(x, \theta, t)$ with the properties (i), (ii), (iii) above. Let $c_{\varepsilon}(x)$ and $\overline{c_{\varepsilon}(x)}$ denote chains obtained from $c(x)$ and $\overline{c(x)}$ respectively by lifting them to $Z$. The lifting should be done in such a way that they belong to the same sheet of $Z$ over the part $\phi_{\varepsilon}(x)$ of $\operatorname{Re} \Omega$ defined by $\varepsilon \varphi(x, \theta)>0$. It is easy to check that this definition is consistent and that the lifted chains will then belong to different sheets over $\phi_{-\varepsilon}(x)$. The construction is also unambiguous apart from a choice of the $\operatorname{sign}$ of $\varphi^{1 / 2}$ at one point (when $U$ is connected).

Definition. A Petrovsky chain for half-integral $q$ is

$$
A(x, q, \varepsilon)=c_{\varepsilon}(x)+\overline{c_{\varepsilon}(x)} \subset Z \backslash \phi(x) .
$$

A Petrovsky cycle is

$$
\alpha(x, q, \varepsilon)=\partial A(x, q, \varepsilon) \subset Z \backslash \phi(x) .
$$

There are also minor misprints as follows. A figure 1.1 means page 1 , line 1 , a figure 1.1 - page 1 , line 1 from the bottom.

53. 15 - read 'contains'. 55.11 - read 'regular'. 56.2 a sign ' $\sim$ ' is missing in the formula (5). 56.7 - read ' $a \rightarrow a^{\prime}$ '. $57.10 \mathrm{read}$ ' $\gamma>0$ '. $57.12 \mathrm{read}$ ' $\gamma=1$ '. $57.8-\operatorname{read}$ ' $p$ !'. $59.15 \mathrm{read}$ ' $-\varphi(x, \theta)$ '. $60.1 \mathrm{read}$ 'manifold'. 60.12 for $k$ read ' $\mu$ '. 61.13 read ' $\partial g / \partial \bar{\theta}$ vanishes of infinite order'. $65.5-\operatorname{read}$ 'Then'. 65.1- $\operatorname{read}$ ' $s \rightarrow$ '. $66.8-\operatorname{read}$ ' $\varphi$ has'.

Received September 21, 1977.

* Lunds University. 